\title{
Survey on Self Driving Vehicle
}

\author{
Harsh Sharma*, Shanky Goyal, Navleen Kaur \\ Department of Information Technology, \\ Chandigarh Engineering College, Landran \\ Email Id: "harsh.4054@cgc.edu.in
}

\begin{abstract}
In this paper we have done survey about the technology of self-driving vehicles also known as autonomous vehicles. Along with this we are going to summarize the future of technology based on benefits and problems. In between the process we also found some importantlevels of this technology. This idea is already implemented in many foreign countries. And it has been proved worthful and successful also. So here, we will discuss about some of the basic info about this technology, levels, impacts, some communication on this topic in other countries along with the final conclusion. That whether it should be implemented more or not and our analysis shows that there is a huge scope in future with the advancementin technology day by day. Finally, it concludes a positive response to this autonomous technology.
\end{abstract}

Indexed Terms- automated, vehicle, driving. (Keywords)

\section{INTRODUCTION}

This self-driving technology mainly deals with the vehicles that can work and movethems elves by sensing the movements and environment without any human presence. These self-driving vehicles are also known as autonomous vehicles. A human driver nor any human passenger is needed compulsorily to operate this vehicle. It totally works on the operations donethrough sensors, remote controlled operations, artificial intelligence. Thus, making it a product of mechatronics field.

A Vehicle that has functionality of autonomous, it means it will aware by itself and has capability to choose by itself. For example, you say "drive me to work", instead to follow your instruction it will be start to drive at another point, no it will not happen, an autonomous vehicle will follow the driver instructions and will reach the destination as driver want.

Sometime, we use words self-driven and autonomous interchangeably but there is minor differencebetween these two. For self-driving vehicle, driver should always be present with in the vehicle, even systemcan drive by itself, but still there should always be driver to take control of vehicle. We divide vehicle into various categories or levels. So self-driving vehicle come under the conditional and high driving automation level. Self-driving vehicles combines a variety of sensors to sense and connect to the nearest sensors, such as radar,LiDAR, sonar, GPS, odometryand inertial measurement units.

This is a future technology that will change our transportation system.it will change the traffic flow on the roads and will also improve the safety of system.Other than that, people who will use such new transportation will also be able to savetheirtime and they can utilize that time for more productivity.it will also us efull for those people that has not capability to utilize the current transportation systemdue to old age, blindness or some other inability.

Many companies are giving their $100 \%$ to achieve this big target and putting their efforts in completing the requisites for deployment of this technology. Research is going on to keep in mind the long term effect of such technology on society and roads.

In this paper, we have focused on motivation of this technology and also the various stages that come during driving. Then in section II literature view of various authors will cover. In Section III, we will discuss various components and levels of the automated vehicle. In Section IV, challenges and befits of automated vehicle are covered.

\section{A. Motivation}

Motivation for automation driving vehicles comes from various perspectives. Few of them are:

> The main motive is always technology advancement in terms of relax ability to human beings, better travel efficiency, save fuel/petrol.

$>$ Next motive is to make world accident free. Based on learning and sceneries, automated vehicle can take decision to avoid accident prone areas and also take immediate safety action, if accident still happens

$>$ The third motivation is to help old aged and visually blinded people who are not able to take advantages of vehicle properly. These automated vehicle help them to provide safe and timely journey

\section{B. Structure of Driving Tasks}

When a human being drives a vehicle, his driving activity involves basically three steps [1]: strategic, tactical and control. Strategic step help human drive to make aplan , tactical step involve human being for taking short term decisions and last step control help them to control the steering[10] based on localized feedback. In automation driving system, all these three task or stages may be used fully or partially at different timescales as explained below:

Strategic task: - basically this step helps to make trip plan that include route, aim and methods through which travelling will happen, based on associated cost, risk and preferences. To make plan, there is requirement of input that is gathered before starting the trip and during the trip. There are basically two type of inputs come to the system-one is through environment and secondly through external interface. Environment provides inputs that are directly observed like weather conditions or visually data. External interfaces are like social media, general information gathering at various time about the route, also provide input to strategic planning.

$>\quad$ Tactic Process: This task involves the basic core processes of driving. At this step, automated system decide basic driving process like what to do at traffic 
light, take a decision for lane change, slow speed if any curve come etc. Traffic management system act like external sources that help automated system to take decisions and directly relevant information like road geometry also work as input at this stage to take controlled action.

Control Level: it take input from tactical level and als o provide back information to tactical level to take decision. Bas ed on object information, it control speed of the vehicle depend on algorithm used [12].

\section{LITERATURE REVIEW}

Cohen, Tom, and Clémence Cavoli [2] have focused on traffic flow control mechanism. Author has told that in future population will increase that will also increase traffic volume and using laissez-fair approach, it will not possible to handle that. Author has provided some interventions that can be used by government at planning, regulatory, infrastructure and services level.

Klomp, Matthijs, et al 「3\ has explained the redundancy required for safety of vehicle.Explanation of brake and steering systemredundancy with fault tolerance is given. Then author has concentrate on path tracking and control mechanism of automated vehicle has been defined.

Cao, Haotian, et al.[4] gives solution for level 2 and level3 driving system.it us es trajectory framework for path planning and speed planning by natural cubic spline and the convexoptimization method. After that it follows MPC approach of linearized time varying and also used vehicle prediction model. Trajectory framework is divided into three layers planning, tactical and control layer. Planning layer provide optimal path, and speed planning, control layer ensure that vehicle should follow planned trajectory and tactical layer take the decision for selection of lane, speed control etc.

Is ermann, R., M. Schorn, and U. Stählin[5] work on project PRORETA which help to avoid collision by automatic functionality of braking and steering. To achieve it, firstly author detects the speed and size of object that are near to the vehicle using LIDAR and video camera. Then calculate a trajectory based on the distant, width and speed of objects. To control it, different methods has used like nonlinear asymptotic output tracking feedback control etc.

Novi, T., et al.[6] has proposed a combination of two nonlinear models for finding the paths. in this control take place at two level: for prediction the break point at lower level, calculate the velocity profile, second level use velocity profile to get velocity constraints[21] which guides the break of the vehicles.

Gora, Pawel, et al[7] has provided review on various microscopic models of Connected and Automated Vehicle. As per author, there are some model which focus on energy efficient strategy, other focus on CASS in different kind of traffic (automated/unautomated), collision free car following model , microscopic models based on lane friction model.
Travis J. Crayton \& Benjamin MasonMeier:These writers summarize there review letter in Highlighted form, the main highlights of their review paper is:

- Autonomous vehicles (AVs) will have consequences across determinants of health.

- Consideration of the public health impacts of AVs has thus far been limited.

- This agenda provides a basis for public health participation in AVpolicymaking.

\section{WORKING AND LEVELS}

In this section, we will discuss technology used for automated vehicle along with the different levels of automations.

\section{A. Components of Automated Vehicle}

The automated vehicles work on software and hardware system combination. Hardware components works like human body and software components works like brain of the body. Its components are broadly classified as follow:

Data Storage: This component is us ed for storage of geographical data, lanes etc.

$>$ Perception: System convert basic information into useful data

$>$ Coordination: helps to coordinate with other objects like vehicles, roads and cloud

$>$ Processing: help toprocess the data and choose the path based on predictable behaviors of other objects.

$>$ Sensors: Sensors help to gather data from various objects like vehicle, video cameras etc.

> Software: it is used to process the data get from various tool and provide useful information to various hardware control components.

\section{B. Working of Automated Vehicle}

Following is the working of different components:

1) For any intelligent vehicle, first step is always to know where they are currently in the world. For that very basic things need to know local coordinates, road boundaries and inters ections, it is local map. Two type of map may be used real time map or stored map. In real time mapmaking, lots of components are use like rndf, gps, camera, lidar, radar etc.

2) Sensor, short range proximity radar, ultrasonic sensors may be used to identify the objects near to vehicle for collision avoidance.

3) For tracking nearby traffic light, some school lane signal or any other signal, track other vehicle can be done by video cameras.

4) To measure distances, detect roadedges,identify lane marking, Lidar (light detection and ranging) can be used.

5) Finally the software part of the automated vehicle system [8] take all this sensory inputs and analyze all that data and provide usable information to vehicle's actuators and send controlled instruction to it. Vehicle actuator controls acceleration, brake and steering [15] using analyzed information that it has received froms oftware. To follow traffic rules and als o to find the obstacle the ways [9], different kind of hard coded rules, different algorithms for obstacle avoidance[13], object recognition and to make different kind of predictions are involved in the software 


\section{Levels of Automated Vehicles}

So, the next main discussion is about the 6 levels that define the automation of vehicles which are explained in table 1[1]:

TABLE1: LEVEL OF AUTOMATIONS

\begin{tabular}{|c|c|c|}
\hline Level & Level Name & Description \\
\hline 0 & No Automation & $\begin{array}{l}\text { At this level, there is no meaning of } \\
\text { automation, complete dependency is } \\
\text { on human driver for handling all kind } \\
\text { of dynamic tasks }\end{array}$ \\
\hline 1 & $\begin{array}{l}\text { Driver } \\
\text { Assistance }\end{array}$ & $\begin{array}{l}\text { Specific tasks like steering or } \\
\text { accelerator is done by the driver } \\
\text { assisted programmed with the } \\
\text { assistance of driver }\end{array}$ \\
\hline 2 & $\begin{array}{l}\text { Partial } \\
\text { Automation }\end{array}$ & $\begin{array}{l}\text { Driver assisted program do both } \\
\text { steering and accelerator task and } \\
\text { human driver do remaining dynamic } \\
\text { tasks }\end{array}$ \\
\hline 3 & $\begin{array}{l}\text { Conditional } \\
\text { Automation }\end{array}$ & $\begin{array}{l}\text { At this level, all dynamic tasks are } \\
\text { done by automated driving system with } \\
\text { driving mode specific performance. } \\
\text { Human driver respond to system as per } \\
\text { its request. }\end{array}$ \\
\hline 4 & $\begin{array}{l}\text { High } \\
\text { Automation }\end{array}$ & $\begin{array}{l}\text { In this type of system, there is no need } \\
\text { of human intervention, all dynamic } \\
\text { tasks are done by automated driving } \\
\text { system but it work in some driving } \\
\text { modes. }\end{array}$ \\
\hline 5 & $\begin{array}{l}\text { Full } \\
\text { Automation }\end{array}$ & $\begin{array}{l}\text { This kind of system work at all } \\
\text { roadways and all kind of } \\
\text { environmental conditions. There is no } \\
\text { need of any kind of human } \\
\text { intervention. }\end{array}$ \\
\hline
\end{tabular}

\section{CHALLENGES}

Testing is already going on various automated vehicles all around the world but still it is not widely available in marked due to various challenges in this concept. These challenges are not just technical but also related to environment also.

Lidar and Radar[19]:To look the surrounding of car to make an automated system, there is need of lot of LiDar and Radar Sensors. These sensors are very expensive and also its processing is totally different to see the object and understands that as compare to emergency breaking an control function of cars [16]

Complex software System: There is need of large amount of complex code to implement an automated system. This systemtake lot of input fromsensor from human and from other information sources and then algorithm applied on this large amount of information to take a right decision. For this complex system, there is need of high end processor also.

Traffic Conditions and Laws: For implementing any automated system, firstly algorithm should be capable to understand our traffic light system [20] and our government laws. Systemshould be aware that what is the rule of traffic light and from how much distance, it should sense the lights.

Pricing: This automated system based vehicles are more costly than as compared to traditional vehicles due to lot of sensors, processor and other components. So, it is not affordable for every person.
Accident Liability: Currently, driver is responsible, if any kind of accident occur. But in case of automated system, there is no liability of driver because he is not driving the car, then who will take responsibility of accidents.

Human intelligence vs Artificial intelligence: While driving[22] a car, drivers read the behavior of the objects that are near to his vehicle through non-verbal communication or through eye contact and based on this data, driver take right decision in less time. But automated system, take decision based on existing knowledge and information captured from sensors. Still, it can't match human sense.

Road Conditions: Automated systems require proper knowledge of lanes. But not everywhere, proper lanes markings are defined. So, it is big challenge to run such systems at that places.

\section{A. Benefits}

Other than challenges, many benefits will get from automated systems that are as follows:

Reduction in Accidents: Automated systems will help to reduce theaccident due to pre knowledge of near objects through sensors.

Less Traffic Congestion: Automated systems will also control[14] the traffic congestion through the knowledge of lanes and less accidents.

Reduced CO2 Emission: if traffic congestion will reduces, automatically $\mathrm{CO} 2$ emission will also reduce

Less consumption of fuel [17]: There is very less consumption of fuel in case of automated system due to reducing distance between the vehicle and road.

Reduce time wastage: it will also help human beings to proper utilize their time in a productive manner. They can save their time in automated vehicles.

\section{CONCLUSION}

There are many challenges in transportation system related to human's life. Because increase in transportation system, also increasing the roads traffic and accidents. There is need of such system which can solve these problems. Automated systems are working on both these issues. Main motive of Self driving system [11] is to provide best facility to humans who are not able to drive thems elves or not physically capable to drive for long time. In this paper, we have tried to cover challenges, benefits, and level components of automated system. Still there is large scope to work on automated system which can help to make it better.

\section{REFERENCES}

[1] Gordon, T. J., and Mathias Lidberg. "Automated driving and autonomous functions on road vehicles." Vehicle System Dynamics 53.7, pp. 958-994, 2015.

[2] Cohen, Tom, and Clémence Cavoli. "Automated vehicles: exploring possible consequences of government (non) intervention for congestion and accessibility." Transport reviews 39.1, pp.129-151, 2019.

[3] Klomp, Matthijs, et al. "Trends in vehicle motion control for automated driving on public roads." Vehicle System Dynamics 57.7, pp.1028-1061, 2019. 
[4] Cao, Haotian, et al. "An optimal hierarchical framework of the trajectory following by convex optimisation for highly automated driving vehicles." Vehicle System Dynamics 57.9,pp. 1287-1317,2019.

[5] Isermann, R., M. Schorn, and U. Stählin. "Anticollision system PRORETA with automatic braking and steering." Vehicle System Dynamics 46.S1, pp. 683-694, 2019.

[6] Novi, T., et al. "Real-time control for at-limit handling driving on a predefined path." Vehicle System Dynamics, pp.1-30, 2019.

[7] Gora, Paweł, et al. "Microscopic traffic simulation models for connected and automated vehicles (CAVs)-state-of-theart." Procedia Computer Science 170 , pp. 474-481, 2020.

[8] Milakis, Dimitris, Bart Van Arem, and Bert Van Wee. "Policy and society related implications of automated driving: A review of literature and directions for future research." Journal of Intelligent Transportation Systems 21.4, pp. 324-348, 2017.

[9] Jonasson, Mats, and M. Thor. "Steering redundancy for selfdriving vehicles using differential braking." Vehicle system dynamics 56.5, pp. 791-809, 2018.

[10] Chen, Liang-Kuang, and A. GalipUlsoy. "Experimental evaluation of a vehicle steering assist controller using a driving simulator." Vehicle System Dynamics 44.3, pp. 223245, 2006.

[11] Cao, Haotian, et al. "An optimal model-based trajectory following architecture synthesising the lateral adaptive preview strategy and longitudinal velocity planning for highly automated vehicle." Vehicle system dynamics 55.8 , pp. 1143-1188, 2017.

[12] Moon, Seungwuk, Wanki Cho, and Kyongsu Yi. "Intelligent vehicle safety control strategy in various driving situations." Vehicle System Dynamics 48.S1, pp 537-554, 2010.

[13] Kim, Young Chol, Kyong-Han Yun, and Kyung-Deuk Min. "Automatic guidance control of an articulated all-wheelsteered vehicle." Vehicle system dynamics 52.4, pp. 456-474, 2014.

[14] Bastian, Andreas. "Fuzzy logic in automatic transmission control." Vehicle System Dynamics 24.4-5, pp 389-400, 1995.

[15] Katzourakis, Diomidis, et al. "Driver steering override strategies for steering based active safety systems." Proceedings of 2nd International Symposium on Future Active Safety Technology towards Zero-Traffic Accidents (FAST-zero'13). 2013.

[16] Rizzi, Matteo, Anders Kullgren, and ClaesTingvall. "Injury crash reduction of low-speed Autonomous Emergency Braking (AEB) on passenger cars." Proc. of IRCOBI Conference on Biomechanics of Impacts. 2014.

[17] Suthaputchakun, Chakkaphong, Zhili Sun, and MehrdadDianati. "Applications of vehicular communications for reducing fuel consumption and CO 2 emission: the state of the art and research challenges." IEEE Communications Magazine 50.12, pp. 108-115, 2012.

[18] Alam, Nima, AsgharTabatabaeiBalaei, and Andrew G. Dempster. "An instantaneous lane-level positioning using DSRC carrier frequency offset." IEEE Transactions on Intelligent Transportation Systems 13.4, pp. 1566-1575, 2012.

[19] Cheng, Wei, et al. "On the design and deployment of RFID assisted navigation systems for VANETs." IEEE Transactions on Parallel and Distributed Systems 23.7, pp. 1267-1274, 2011.

[20] Xu, Yang, Yulin Zhang, and Ming Liu. "Multiagent based decentralized traffic light control for large urban transportation system." Mathematical Problems in Engineering, 2014

[21] Kim, Tae Soo, Chris Manzie, and Rahul Sharma. "Model predictive control of velocity and torque split in a parallel hybrid vehicle." 2009 IEEE International Conference on Systems, Man and Cybernetics.IEEE, 2009.

[22] Schleicher, Susanne, and ChristhardGelau. "The influence of Cruise Control and Adaptive Cruise Control on driving behaviour-A driving simulator study." Accident Analysis \& Prevention 43.3, pp.1134-1139, 2011. 